\title{
因HAD
}

ISSN-L: 2530-5115

(1) (1)(2)

DOI: http://doi.org/10.22585/hospdomic.v3i3.79

\section{Eficacia y seguridad de la infusión subcutánea de furosemida en domicilio para el manejo de la insuficiencia cardiaca}

Efficacy and safety of subcutaneous furosemide infusion at home for the management of heart failure

Aarón Gutiérrez Pastor', Rafael López-Bas Valero², Annabella Freitas Amorós' 1. Hospital San Juan de Alicante. Unidad de Hospitalización a Domicilio. Sant Joan d'Alacant, España.

2. Médico de Hospitalización a Domicilio.

Correspondencia/Correspondence

Aarón Gutiérrez Pastor

aaron.gutierrez.pastor@hotmail.com

Recibido/Received

10.06.2019

Aceptado/Accepted

14.06.2019
Conflicto de Intereses/Competing interest Los autores y autora del trabajo declaran no tener ningún conflicło de interés.

CÓMO CITAR ESTE TRABAJO | HOW TO CITE THIS PAPER

Gutiérrez Pastor A, López-Bas Valero R, Freitas Amorós A. Eficacia y seguridad de la infusión subcutánea de furosemida en domicilio para el manejo de la insuficiencia cardiaca. Hosp Domic. 201 9;3(3):225-32 


\section{RESUMEN}

La Insuficiencia cardiaca es una patología muy prevalente, siendo una de las principales causas de morbi-mortalidad en países desarrollados. Su tratamiento se basa, entre otros fármacos, en la administración de diuréticos para mejorar la sintomatología del paciente. Sin embargo, en muchas ocasiones, la falta de mejoría con el tratamiento oral supone la necesidad de ingreso hospitalario para la administración por vía intravenosa.

Presentamos dos casos de pacientes ingresados a cargo de la Unidad de Hospitalización a Domicilio que presentaban insuficiencia cardiaca refractaria a pesar de tratamiento con diuréticos por vía oral. Se inició infusión de furosemida subcutánea en ambos. En el primer caso, presentó mejoría de la sintomatología con resolución del cuadro. En el segundo, al tratarse de un paciente en situación terminal, se produce una mejoría de la clínica pero, debido a su situación, se decidió no completar el tratamiento.

Palabras clave: Insuficiencia cardiaca; Edema; Furosemida; Servicios de Atención a Domicilio Provisto por Hospital.

\section{ABSTRACT}

Heart failure is a very prevalent pathology, being one of the main causes of morbidity and mortality in developed countries. The treatment is based, among other drugs, on the administration of diuretics to improve the patient's symptoms. However, in many cases, the lack of improvement with oral medication implies the need for hospital admission for intravenous administration.

We present two cases of patients admitted to the Hospital at Home Unit who had refractory heart failure despite adequate diuretic treatment. Subcutaneous furosemide infusion was initiated in both. The first case presented symptom improvement with clinical resolution. The second was a terminally ill patient. Initially there was a clinical improvement but, due to the situation, the final decision was not to complete the treatment.

Keywords: Heart Failure; Edema; Furosemide; Home Care Services, Hospital-Based. 


\section{INTRODUCCIÓN}

La Insuficiencia cardiaca (IC) es un síndrome caracterizado por una incapacidad del corazón para bombear sangre en la cantidad para suplir los requerimientos metabólicos del organismo.

En los países desarrollados la prevalencia se sitúa en torno al 2\% en la población mayor a 40 años. En España, esta cifra es mayor, oscilando entre un 5-7\% en función de los estudios, sin encontrar diferencias entre sexos. Sin embargo, la prevalencia de la IC aumenta exponencialmente con la edad, pudiendo alcanzar cifras superiores al 18\% en los mayores de 75 años (1).

Además, según un estudio de la Sociedad Europea de Cardiología (ESC) se espera un incremento sostenido en la prevalencia de IC en los próximos años, pudiendo alcanzar cifras de un $25 \%$ en el 2030 (1). Este aumento puede explicarse por varias razones como el envejecimiento progresivo de la población, el aumento de los factores de riesgo, la eficacia de la prevención, así como la mayor supervivencia de pacientes.

Las enfermedades cardiovasculares (ECV) suponen la primera causa de mortalidad en los países desarrollados. Dentro de ellas, Ia IC es la tercera causa de mortalidad cardiovascular, tras la cardiopatía isquémica y la enfermedad cerebrovascular. En España, según datos del INE, las ECV fueron la primera causa de mortalidad femenina (270,5 muertes por cada 100.000) y la segunda entre los varones $(233,2)$ en el año 2015 (2).

La mortalidad hospitalaria de los pacientes con IC es del 4-7\%, pudiendo aumentar hasta el 7-11\% entre los 60-90 días posteriores. Además, el porcentaje de reingresos en este tiempo se sitúa entre el 25-30\%. En cuanto a las causas, el 65,5\% de las muertes por IC fueron de causa cardiovascular, predominantemente por progresión de la IC (el 32,2\% del total de muertes), seguida de la muerte súbita (16\%), el infarto agudo de miocardio $(8,3 \%)$ y otras causas cardiovasculares $(5,1 \%)$. El 9,8\% falleció por una causa no cardiovascular (2).

Dentro del tratamiento de la IC, los diuréticos, aunque no modifican la enfermedad, constituyen un papel importante para el tratamiento de los pacientes con IC y signos de sobrecarga de fluidos y congestión. Concretamente, son útiles en el manejo de la disnea y edema periférico; así como para mantener la euvolemia (3).

Su efecto se basa en el aumento de la secreción renal de agua y sal; presentando, además, cierto efecto vasodilatador. Se recomiendan en todos los pacientes con IC aguda y signos de sobrecarga, evitando aquellos casos de IC aguda con signos de hipoperfusión (4).

En la IC, la furosemida intravenosa es el diurético más empleado. Sin embargo, la evidencia científica actual en apoyo de esta práctica es escasa. Se debe limitar la dosis a la menor cantidad necesaria para lograr un efecto clínico adecuado; así como modificarla según la función renal y las dosis de diuréticos previas.

En la actualidad, no existe un régimen óptimo de tratamiento en cuanto a la dosis, tiempo o vía de administración. Se han llevado a cabo diferentes revisiones y metaanálisis sobre la diferencia en cuanto a seguridad y eficacia de la infusión continua frente a la inyección de bolos seriados, sin encontrar diferencias significativas (4).

Además, se ha observado que, a medida que avanza la enfermedad, puede aparecer una insuficiencia cardiaca refractaria, en la que persisten los síntomas que limitan la vida diaria (clase funcional III O IV) a pesar del tratamiento óptimo con los fármacos de eficacia probada (5). Ésta puede afectar hasta a un $25 \%$ de los pacientes, lo que supone un reto en el tratamiento de la IC, pues la persistencia de datos congestivos se asocia a mayor mortalidad (6).

Ante esta resistencia, las investigaciones actuales se centran en la búsqueda de diferentes medidas terapéuticas como son la combinación de distintas clases de diuréticos para conseguir un efecto sinérgico, la infusión de suero salino hipertónico, etc. 
Una de las más recientes es el empleo de la vía subcutánea para la administración de Furosemida, bien de forma tradicional o mediante bombas elastoméricas, sobre todo a nivel de los cuidados paliativos y, recientemente, en Unidades de Insuficiencia Cardiaca. Se ha demostrado que su efecto en voluntarios sanos se inicia a los 30 minutos del inicio de la infusión, algo más tarde que si se administra de forma intravenosa. Sin embargo, consigue aumentar el volumen urinario y presenta una duración del efecto de unas 4 horas (7).

La Furosemida subcutánea supone un avance para el tratamiento sintomático de la IC, pues permite la administración en diferentes medios, reduciendo ingresos y visitas hospitalarias (8), y suponiendo una mayor confortabilidad para el paciente.

Sin embargo, en la actualidad, la utilización de Furosemida por vía subcutánea no presenta ensayos controlados y aleatorizados en la población de tratamiento y, además, los datos de observación se derivan de cohortes pequeñas (9). Este hecho supone que, a nivel de cuidados paliativos, se prescriba fuera de ficha técnica.

\section{DESARROLLO DE LA EXPERIENCIA}

Se presentan dos casos clínicos. El primero, es el de una mujer de 78 años de edad con antecedentes de hipertensión arterial, hipertensión pulmonar, enfermedad pulmonar obstructiva crónica e insuficiencia cardiaca congestiva. Es parcialmente dependiente para las actividades básicas de la vida diaria, y portadora de oxígeno 24 horas y BIPAP (siglas en inglés de "presión positiva continúa en la vía aérea") nocturna. Es remitida desde Atención Primaria a la Unidad de Hospitalización a Domicilio de San Juan de Alicante por insuficiencia cardiaca descompensada con presencia de edemas bilaterales hasta raíz de miembros y crepitantes en ambas bases pulmonares a pesar de la administración de Furosemida por vía oral.

Ante la clínica de la paciente y la dificultad para la canalización de vías periféricas eficientes para el tratamiento de la paciente, se inicia perfusión de furosemida (180 mg/día) con infusor por vía subcutánea a $1 \mathrm{ml} / \mathrm{h}$ durante 5 días.

Tras 15 días de tratamiento, se observa una mejoría del cuadro clínico, con reducción de edemas en miembros inferiores, ausencia auscultatoria de crepitantes a nivel pulmonar y posibilidad de inicio de la deambulación.

Figura 1. Lugar de inserción de la perfusión al inicio del tratamiento

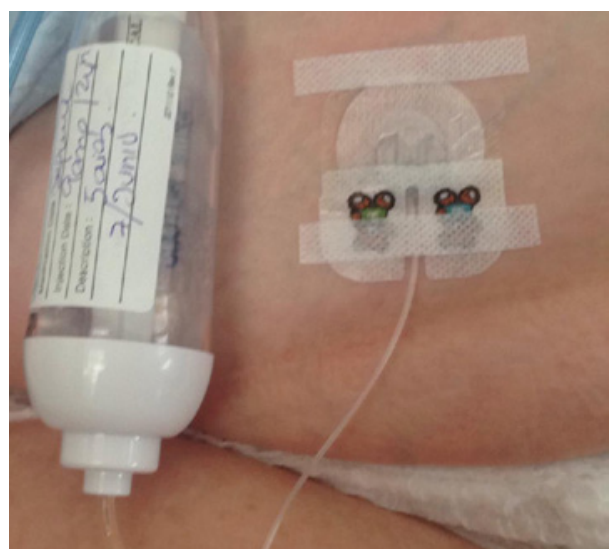

Figura 2. Lugar de inserción de la perfusión al final del tratamiento

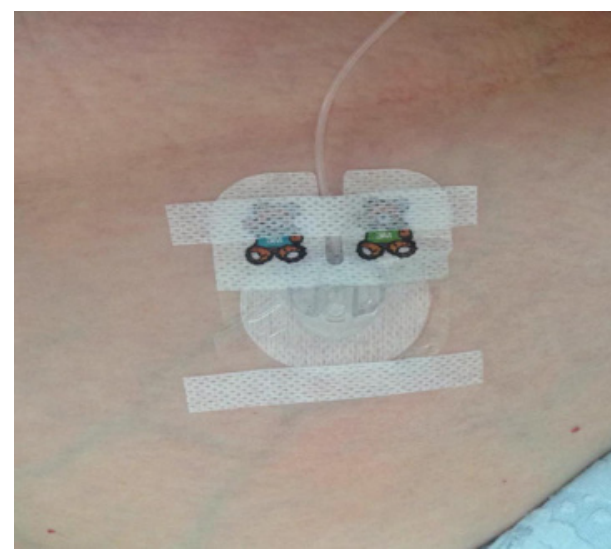


Además, y a pesar de tratarse con Furosemida subcutánea pH alcalino, la paciente no ha presentado ningún efecto secundario ni a nivel local ni sistémico (Figuras 1-2).

Realizamos seguimiento clínico y analítico de la paciente, cuyos resultados aparecen reflejados en la tabla 1.

Tabla 1. Mediciones y controles analíticos Paciente 1

\begin{tabular}{|c|c|c|c|c|}
\hline & $\begin{array}{l}{ }^{\text {er día }} \\
\text { (Basal) }\end{array}$ & $\begin{array}{l}5^{\circ} \text { día de } \\
\text { tratamiento }\end{array}$ & $\begin{array}{l}10 \text { día de } \\
\text { tratamiento }\end{array}$ & $\begin{array}{l}14^{\circ} \text { día de } \\
\text { tratamiento }\end{array}$ \\
\hline $\begin{array}{l}\text { Tobillo derecho } \\
(\mathrm{cm})\end{array}$ & 25 & 24 & 24 & 24 \\
\hline $\begin{array}{l}\text { Tobillo } \\
\text { izquierdo }(\mathrm{cm})\end{array}$ & 25 & 24 & 24 & 25 \\
\hline $\begin{array}{l}\text { Pantorrilla } \\
\text { derecha }(\mathrm{cm})\end{array}$ & 39 & 38 & 37 & 38 \\
\hline $\begin{array}{l}\text { Pantorrilla } \\
\text { izquierda }(\mathrm{cm})\end{array}$ & 38 & 39 & 39 & 39 \\
\hline $\begin{array}{l}\text { Muslo derecho } \\
(\mathrm{cm})\end{array}$ & 57 & 53,5 & 53 & 48 \\
\hline $\begin{array}{l}\text { Muslo izquierdo } \\
(\mathrm{cm})\end{array}$ & 58 & 56 & 53 & 53 \\
\hline $\begin{array}{l}\text { Sensación } \\
\text { disneica }(1-10)\end{array}$ & 10 & 7 & 5 & 3 \\
\hline $\begin{array}{l}\text { Diuresis } \\
\text { (ml/24h) }\end{array}$ & 1800 & 2150 & 2400 & 2600 \\
\hline $\mathrm{TA}(\mathrm{mmHg})$ & $125 / 65$ & $110 / 50$ & $115 / 60$ & $135 / 65$ \\
\hline $\mathrm{Na}+(\mathrm{mmol} / \mathrm{L})$ & 147 & 142 & 145 & 144 \\
\hline $\mathrm{K}+(\mathrm{mmol} / \mathrm{L})$ & 3,3 & 2,9 & 3,1 & 3,1 \\
\hline $\begin{array}{l}\text { Creatinina (mg/ } \\
\mathrm{dL} \text { ) }\end{array}$ & 1,01 & 0,89 & 0,8 & 0,82 \\
\hline $\mathrm{FG}(\mathrm{mL} / \mathrm{min})$ & 52,81 & $>60$ & $>60$ & $>60$ \\
\hline
\end{tabular}

El segundo caso, se trata de un varón de 84 años de edad con antecedentes de insuficiencia cardiaca refractaria en situación paliativa terminal. Presenta hipertensión arterial, fibrilación auricular, insuficiencia renal crónica y Púrpura Trombocitopénica inmune. Además, había sido intervenido 
de columna por discopatía y estenosis de canal a nivel de L3-L4, por lo que es parcialmente dependiente para las actividades básicas de la vida diaria. Ha sido remitido a la Unidad de Hospitalización a Domicilio de San Juan de Alicante por edema a nivel de miembro inferior derecho de una semana de evolución.

Ante la clínica del paciente, se inicia perfusión de Furosemida (120mg/día) con infusor por vía subcutánea a $1 \mathrm{ml} / \mathrm{h}$ durante 5 días. Tras 8 días de tratamiento, se observa una mejoría del cuadro clínico, con reducción del edema en el miembro inferior. Sin embargo, el paciente se encuentra con un empeoramiento de su estado basal, con disminución de respuesta a estímulos e ingesta. Debido a ello, se consensua con la familia la limitación del esfuerzo terapéutico.

Realizamos seguimiento clínico y analítico de la paciente, cuyos resultados aparecen reflejados en la tabla 2 y 3.

Tabla 2. Controles analíticos Caso 2

\begin{tabular}{|l|l|l|}
\hline & $\mathbf{1}^{\text {er }}$ día (Basal) & $\mathbf{7}^{\circ}$ día de tratamiento \\
\hline Creatinina & 1,8 & 2,29 \\
\hline Filtrado Glomerular & 36,07 & 27,32 \\
\hline $\mathrm{Na}^{+}$ & 136 & 148 \\
\hline $\mathrm{K}^{+}$ & 3,80 & 3,5 \\
\hline
\end{tabular}

Tabla 3. Mediciones diuresis Caso 2

\begin{tabular}{|l|l|l|l|l|l|l|l|}
\hline Día de tratamiento & $1^{\circ}$ & $2^{\circ}$ & $3^{\circ}$ & $4^{\circ}$ & $5^{\circ}$ & $6^{\circ}$ & $7^{\circ}$ \\
\hline Diuresis (ml) & 1700 & 2600 & 1850 & 1700 & 1550 & 950 & 1000 \\
\hline
\end{tabular}

\section{CONCLUSIONES}

En la actualidad, los avances en el diagnóstico y tratamiento de la insuficiencia cardiaca han permitido una mejora en la supervivencia y la calidad de vida de los pacientes, con una reducción, además, de los ingresos hospitalarios. Sin embargo, el manejo de la congestión en pacientes ambulatorios con IC continúa siendo un problema.

En ocasiones, el uso de diuréticos orales, tanto en monoterapia como en combinación, no permite la resolución del cuadro. Por este motivo, puede ser necesaria la administración de furosemida intravenosa, lo que conlleva, generalmente, el ingreso hospitalario.

Sin embargo, la utilización de la vía subcutánea para la administración de furosemida, tanto de forma tradicional como mediante elastómeros, ha permitido reducir el número de estos ingresos. 
No obstante, el pH alcalino limita la tolerabilidad a largo plazo en algunos pacientes; motivo por el que, además, se ha desarrollado una Furosemida con pH neutro cuyos resultados son similares a la vía intravenosa en cuanto a eficacia y seguridad (10).A pesar de ello, en el caso de nuestros dos pacientes se ha observado una eficacia del tratamiento por vía subcutánea con mejoría de la clínica y situación de ambos. Además, y a pesar de que determinados estudios limitan la tolerabilidad de esta vía, nuestros pacientes no han presentado efectos secundarios, ni a nivel local ni sistémico.

Debido a ello, concluimos que, la furosemida subcutánea puede ser un medio práctico y rentable para proporcionar una terapia diurética con asa parenteral en una población frágil con insuficiencia cardiaca avanzada y en las que las otras formulaciones no son posibles. Sin embargo para la generalización de ésta sería necesario estudios más sólidos y estandarizados que prueben esta hipótesis.

\section{BIBLIOGRAFÍA}

1. Sayago-Silva I, García-López F, Segovia-Cubero J. Epidemiology of heart failure in Spain over the last 20 years. Rev Esp Cardiol (Engl Ed). 2013;66(8):649-56. DOI: 10.1016/j.rec.2013.03.012; PMID: 24776334

2. Gómez-Martínez L, Orozco-Beltrán D, Quesada JA, Bertomeu-González V, Gil-Guillén VF, López-Pineda A, Carratalá-Munuera C. Tendencias de mortalidad prematura por insuficiencia cardiaca por comunidades autónomas en España, periodo 1999-2013. Rev Esp Cardiol. 2018;71:531-7. DOI: 10.1016/j.recesp.2017.09.014

3. Krzysztofik J, Ponikowski P. Current and emerging pharmacologic options for the management of patients with chronic and acute decompensated heart failure. Expert Rev Clin Pharmacol. 2017;10(5):517-534. DOI: 10.1080/17512433.2017; PMID: 28358228

4. Jenkins PG. Diuretic strategies in patients with acute heart failure. N Engl J Med. 2011;364(21): 2066. DOI:10.1056/NEJMc1103708; PMID: 21612482

5. Hunt S, Backer D, Chin M, Cinquegrani M, Feldman A, Francis G, et al; ACC/AHA guidelines for the evaluation and management of chronic heart failure in the adult: executive summary: $A$ report of the american college of cardiology/american heart association task force on practice guidelines (committee to revise the 1995 guidelines for the evaluation and management of heart failure) developed in collaboration with the international society for heart and lung transplantation endorsed by the heart failure society of america. J Am Coll Cardiol 2001;38(7):2101-13. DOI: 10.1016/S0735-1097(01)01683-7

6. Sánchez E, Rodríguez C, Ortega T, Díaz-Molina B, García-Cueto C. Tratamiento de la sobrecarga de volumen en la insuficiencia cardiaca refractaria. Insuf Card 2011;6(1):19-26.

7. Gallardo Avilés R, Gamboa Antiñolo F. Monografía SECPAL № 4: Uso de la vía subcutánea en cuidados paliativos [monografía en Internet]. Madrid, España: Sociedad Española de Cuidados Paliativos; 2013 [consultada 13 junio 2019]. Disponible en: http://bit.ly/2ZgwSro

8. Zatarain-Nicolás E, López-Díaz J, de la Fuente-Galán L, García Pardo H, Recio-Platero A, San Román-Calvar JA. Tratamiento de la insuficiencia cardiaca descompensada con furosemida subcutánea mediante bombas elastoméricas: experiencia inicial. Rev Esp Cardiol. 2013;66(12):1002-4. DOI: 10.1016/j.recesp.2013.06.010 
Notas Clínicas: Gutiérrez Pastor, López-Bas Valero, Freitas Amorós

9. Beattie JM, Johnson MJ. Subcutaneous furosemide in advanced heart failure: has clinical practice run ahead of the evidence base? BMJ Support Palliat Care. 2012;2(1):5-6. DOI: 10.1136/ bmjspcare-2011-000199; PMID: 24653487

10. Gilotra NA, Princewill O, Marino B, Okwuosa IS, Chasler J, Almansa J, et al. Efficacy of intravenous furosemide versus a novel, ph-neutral furosemide formulation administered subcutaneously in outpatients with worsening heart failure. JACC Heart Fail. 2018;6(1):65-70. DOI: 10.1016/j.jchf.2017.10.001; PMID: 29226816 